\title{
Perfil Epidemiológico e Qualidade de Vida dos Estudantes de Medicina Portadores de Hiperidrose Primária
}

\author{
Epidemiological Profile and Quality of \\ Life of Medicine Students with Primary \\ Hyperhidrosis
}

\author{
Sônia Oliveira Limal \\ Maria Tainar Barbosa de Almeida ${ }^{I \oplus}$ \\ Fernanda Rodrigues Rocha \\ Rafael Silva Santos ${ }^{l}$ \\ Renata Lima Batalha de Andrade \\ Carla Viviane Freitas de Jesus ${ }^{I}$
}

PALAVRAS-CHAVE

- Hiperidrose.

- Qualidade de vida

- Estudante.

- Saúde.

- Medicina.

\section{RESUMO}

Hiperidrose é a produção excessiva de suor pelo corpo, podendo ser primária ou secundária e generalizada ou localizada. As regiões craniofacial, axilar, palmar, dorso, face interna das coxas, plantar são comumente afetadas. A hiperidrose primária (HP), embora seja uma doença benigna, apresenta um eminente impacto negativo na qualidade de vida do paciente. Essa doença é classificada quantitativamente de acordo com a Escala de Gravidade da Doença da Hiperidrose, com base em como ela pode afetar as atividades diárias, podendo ser de leve a grave intensidade. Os acadêmicos de medicina enfrentam situações desgastantes físicas e emocionais durante seus afazeres e, quando portadores de doenças crônicas como a HP, podem ser estigmatizados como inseguros na sua profissão. Objetivou-se definir a prevalência, os critérios diagnósticos, a gravidade e o impacto na qualidade de vida que a HP proporciona nos âmbitos profissional e extracurricular dos acadêmicos de medicina. Trata-se de um estudo transversal de abordagem quantitativa de caráter descritivo, que inclui 300 acadêmicos do curso de Medicina da Universidade Tiradentes, em Aracaju/SE, no período de agosto a novembro de 2017. Utilizaram-se frequências absolutas e relativas no caso de variáveis categóricas e medidas de tendência e variabilidade central no caso de variáveis numéricas. O valor de $p<0,05$ foi considerado significativo. A prevalência de HP em estudantes de medicina foi de $18 \%$ sem diferença entre os sexos e com predomínios das cores branca e parda. A doença ocorreu principalmente em sítios combinados, como palmoplantar, sendo a região palmar a mais acometida. Os sintomas iniciaram-se, principalmente, durante a infância e adolescência, com prejuízo importante nas atividades diárias pessoais e laborativas, e exacerbação do suor com situações de estresse. A gravidade da HP mais encontrada foi a moderada, sendo o impacto negativo na qualidade de vida principalmente relatado nos graus mais avançados da doença, dificultando as atividades acadêmicas. É importante a inclusão do conhecimento da HP no currículo médico para uma maior divulgação da doença, de modo a permitir um diagnóstico precoce e relacionado à intensidade da sudorese na sua abordagem terapêutica. 


\section{KEY-WORDS}

- Hyperhidrosis.

- Quality of life.

- Student.

- Health.

- Medicine.

\begin{abstract}
Hyperhidrosis is the excessive production of sweat by the body, which can be primary or secondary and generalized or localized. The craniofacial, axillary, palmar, dorsum, inner thigh, and plantar regions are commonly affected. Primary hyperhidrosis (HP), although a benign disease, has an imminent negative impact on the patient's quality of life. This disease is quantitatively classified according to the Hyperhidrosis Disease Severity Scale, based on how the disease affects daily activities, ranging from mild to severe intensity. Medical undergraduate students face physical and emotional stressful situations during their tasks, and when they have a chronic illness such as HP, they can be stigmatized as unsafe for their profession. The objective was to define HP prevalence, diagnostic criteria, severity and impact on the quality of life in the professional and extracurricular scenario of undergraduate medical students. This is a cross-sectional quantitative study of a descriptive nature, including 300 students from the Tiradentes University medical school, in Aracaju-SE, from August to November 2017. Absolute and relative frequencies were used in the case of categorical variables and measures of central tendency and variability were used for numerical variables; $p$ value was considered significant when $p<0.05$. The prevalence of HP in medical students was $18 \%$, with no difference between the genders and with the predominance of white and brown ethnicities. The disease occurred mainly in combined sites, such as palmar-plantar, with the palmar region being the most affected one. The symptoms began mainly during childhood and adolescence, with significant impairment in daily personal and work activities, with disease exacerbation under stress situations. The most common HP severity was moderate, with a negative impact on quality of life, mainly reported at the more advanced stages of the disease, hindering academic activities. It is important to include the knowledge of HP in the medical curriculum for a greater dissemination of information on the disease, allowing its early diagnosis and related to the intensity of sweating in its therapeutic approach.
\end{abstract}

Recebido em: 7/7/19

Aceito em: $17 / 9 / 19$

\section{INTRODUÇÃO}

Hiperidrose é a produção excessiva de suor pelo corpo, além do exigido pelo meio ambiente e do requerido para a homeostase do organismo. Essa doença pode ser primária ou secundária e generalizada ou localizada ${ }^{1}$. No caso da hiperidrose primária (HP), o suor ocorre devido a uma hiperatividade do tronco simpático, sem nenhuma causa relacionada a doenças como hipertireoidismo, obesidade, distúrbio psiquiátrico ou menopausa ${ }^{2,3}$. A HP pode manifestar-se isoladamente nos sítios craniofacial, axilar, palmar e plantar, no dorso e na face interna das coxas. Pode haver associações entre os sítios ou concomitantemente nessas regiões ${ }^{4,5}$.

A HP é classificada da seguinte forma: grau leve (área molhada sem gotejar), moderada (área molhada e gotejante) ou intensa (área molhada e muito gotejante) ${ }^{6-8}$. A HP é classificada qualitativamente de acordo com a Escala de Gravidade da Doença da Hiperidrose (Hyperhidrosis Disease Severity Scale - HDSS), com base em como a doença pode afetar as atividades diárias. $\mathrm{O}$ paciente seleciona a declaração que melhor reflete sua experiência com transpiração na área especificada. Uma pontuação de 3 ou 4 indica hiperidrose grave, enquanto uma pontuação de 1 ou 2 indica HP leve ou moderada9.

A HP apresenta uma prevalência muito variável, de $0,6 \%{ }^{10}$ a $16,8 \%^{11}$, e tem início, principalmente, na infância e adolescência ${ }^{12-15}$. As regiões mais acometidas são palmar, plantar e axilar, gerando prejuízo nas atividades diárias ${ }^{9,16-19}$. A HP, embora seja uma doença benigna, apresenta um eminente impacto negativo na qualidade de vida $(\mathrm{QdV})$ do paciente por causar limitações ocupacionais, educacionais, na interação social, nas atividades físicas e no lazer, trazendo consigo distúrbios psicológicos e de relacionamento ${ }^{1,20}$.

Diversos estudos têm utilizado medidas de QdV para avaliar a profundidade do seu impacto nos portadores de HP. Após a criação do questionário validado por Campos et al. ${ }^{4}$, este passou a ser utilizado nas pesquisas, o que permitiu a padronização na interpretação da avaliação da QdV em pacientes portadores dessa afecção. Mesmo que a hiperidrose não seja considerada uma doença grave, é acompanhada por um 
impacto negativo na QdV dos seus portadores e, por ser crônica, tem enfoque psicológico, físico, social, profissional, além comprometer as atividades de lazer ${ }^{3,20}$.

Observa-se um número crescente de estudos utilizando questionários que avaliam o impacto na $\mathrm{QdV}$ relacionados a várias doenças clínicas e cirúrgicas. Avaliar o impacto na QdV traz elementos comparativos antes e após determinado tratamento e também quantitativos com escore de $Q \mathrm{dV}^{4,3}$. Essa avaliação permite estimar a multidimensionalidade das relações da HP nos vários aspectos pessoais, sociais e profissionais. Pesquisas sobre QdV sugerem que a magnitude dos efeitos adversos provocados por HP é comparável a outras condições, como psoríase grave, insuficiência renal em estágio final e artrite reumatoide ${ }^{21}$.

Essa afecção estigmatizante provoca dificuldades nas atividades do dia a dia dos seus portadores, especialmente aqueles que utilizam manobras manuais. Ainda permanecem sem evidência os impactos na QvD dos portadores de HP nessa população. Diante do que foi apresentado, considerando que a atividade laboral do estudante de medicina depende de destreza manual e da interação com outros profissionais, objetivou-se compreender a prevalência, os critérios diagnósticos, a gravidade e o impacto na QdV que a HP provoca nos âmbitos profissional e extracurricular dos acadêmicos de medicina.

\section{METODOLOGIA}

Trata-se de um estudo transversal de abordagem quantitativa de caráter descritivo. A população em estudo foi do tipo finita, composta pelos discentes dos cursos de Medicina de uma universidade particular de Aracaju/SE, matriculados na instituição até o segundo semestre de 2017. O estudo foi realizado no período de agosto a novembro de 2017. De acordo com informações coletadas disponibilizadas pela coordenação do curso, o quantitativo de acadêmicos matriculados foi de 548. O cálculo da amostra segue o descrito na equação realizada pela fórmula de Barbetta ${ }^{22}$. Foram calculados intervalos de confiança (IC) a 95\% para todas as estimativas, tendo como parâmetro o erro amostral de 0,05.

De acordo com a fórmula, a amostra mínima calculada foi de 232 acadêmicos e a estudada totalizou 300 acadêmicos, selecionados de forma aleatória e sistemática pelo programa estatístico BioEstat 5.0, de maneira a garantir uma amostra homogênea. A pesquisa foi realizada de acordo com as diretrizes e normas regulamentadoras de pesquisa com seres humanos e atende à Resolução no 466, de 12 de dezembro de 2012, do Conselho Nacional de Saúde do Ministério de Saúde. O estudo foi aprovado pelo Comitê de Ética e Pesquisa e protocolado com o número do parecer 260511.
Para a triagem dos acadêmicos portadores de hiperidrose entre a população estudada, foi aplicado o questionário proposto por Felini, Demarchi, Fistarol, Matiello e Delorenze ${ }^{13}$, que define critérios objetivos para definição diagnóstica. Os indivíduos que obtiveram resultado positivo segundo os critérios abordados nessa etapa responderam ao questionário com dados sobre o impacto da HP nas atividades acadêmicas e extracurriculares dos estudantes e o perfil sociodemográfico. Esse questionário foi composto por variáveis inerentes à identificação, principais atividades prejudicadas pela HP.

Em seguida, os acadêmicos portadores de HP preencheram um terceiro questionário com o objetivo de complementar a avaliação da QdV. Esse instrumento foi validado e descrito por Amir, Arish, Weinstein, Pfeffer e Levy ${ }^{23}$ e modificado por Campos et al. ${ }^{4}$. Esse instrumento avalia 20 atividades sobre quatro domínios: funcional-social, pessoal, emocional e condições especiais. $\mathrm{O}$ escore total do questionário varia de 20 a 100 pontos e é obtido pela soma da avaliação de cada atividade, sendo classificadas em cinco níveis de satisfação. Dessa forma, os subescores são classificados, segundo Campos et al. ${ }^{4}$, assim: satisfação muito ruim - acima de 84; ruim - de 68 a 83; boa de 52 a 67; muito boa - de 36 a 51; excelente - de 20 a 35.

Os questionários utilizados apresentam validação no Brasil e internacionalmente. Os dados coletados nesta pesquisa foram inseridos em uma planilha eletrônica no Excel 2016. A análise estatística dos dados utilizou o programa BioEstat 5.0, e a análise descritiva das variáveis foi apresentada por frequência, tendência, variabilidade numérica e desvio padrão, complementada pelos testes Shapiro-Wilk, Anova, Kruskal-Wallis e Pearson. Considerou-se significativo valores de $p<0,05$.

\section{RESULTADOS}

Em um total de 300 acadêmicos que responderam aos questionários, 106 (35\%) eram do sexo masculino e 194 (65\%) do feminino; e 54 (18\%) preencheram os critérios diagnósticos para HP. Dos 54 acadêmicos, 19 (35,2\%) foram do sexo masculino e $35(64,8 \%)$ do feminino, o que corresponde a uma prevalência de $17,9 \%$ nos homens e $18,1 \%$ nas mulheres. Nesse total, 3 $(5,5 \%)$ se declararam negros, $19(35,2 \%)$ pardos e $31(57,4 \%)$ brancos; um acadêmico não declarou sua cor. A média da idade foi de 21,57 anos (Figura 1).

Sobre as características da doença, 18 (33,3\%) estudantes de medicina referiram início dos sintomas da HP na infância, $32(59,2 \%)$ na adolescência e $4(7,4 \%)$ na fase adulta.

Em relação à frequência dos critérios diagnósticos dos acadêmicos portadores de HP, 43 (79,6\%) referiram suor bilateral e relativamente simétrico, 38 (70,4\%) apresentaram frequência de pelo menos um episódio de suor excessivo por semana, 14 


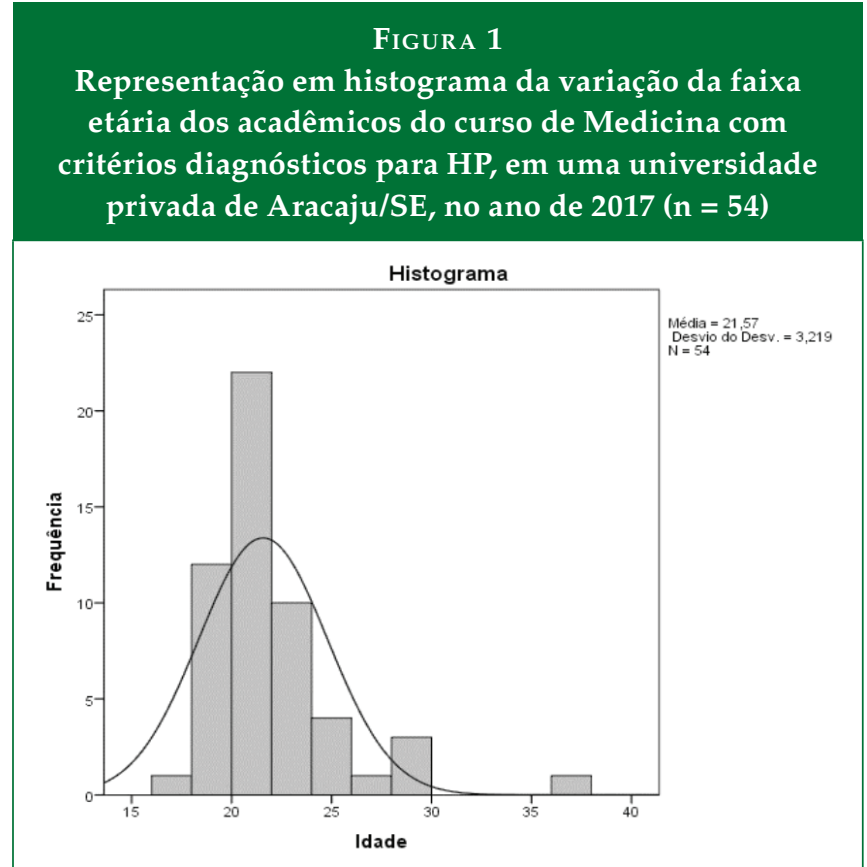

Fonte: Elaborada pelos autores.

(25,9\%) relataram prejuízo nas atividades diárias, 41 (75,9\%) apresentaram os sintomas com idade inferior a 25 anos, 11 $(20,4 \%)$ apresentaram história familiar positiva, 36 (66,7\%) referiram piora dos sintomas com o estresse e 15 (27,8\%) não relacionaram a sudorese com a interferência de temperatura.

A principal área afetada foi a associação das regiões palmar e plantar em 15 (27,80\%) acadêmicos, seguida da região axilar isolada em 10 (18,52\%) acadêmicos, da associação das regiões palmar, plantar e axilar em 7 (12,96\%) acadêmicos e em outras regiões nos demais. De modo geral, a região palmar foi a área mais citada: 35 (64,83\%) alunos (Tabela 1).

\begin{tabular}{|c|c|}
\hline \multicolumn{2}{|c|}{$\begin{array}{c}\text { TABELA } 1 \\
\text { Frequência das áreas afetadas pela HP em } \\
\text { acadêmicos do curso de Medicina portadores } \\
\text { da doença em uma universidade privada de } \\
\text { Aracaju/SE, no ano de } 2017(\mathrm{n}=54)\end{array}$} \\
\hline ÁREAS AFETADAS & FREQUÊNCIA \\
\hline Palmar + Plantar & $15(27,80 \%)$ \\
\hline Axilar & $10(18,52 \%)$ \\
\hline Palmar + Plantar + Axilar & $7(12,96 \%)$ \\
\hline Palmar & $5(9,26 \%)$ \\
\hline Palmar + Axilar & $5(9,26 \%)$ \\
\hline Facial & $2(3,70 \%)$ \\
\hline Axilar + Facial & $2(3,70 \%)$ \\
\hline Dorsal & $1(1,85 \%)$ \\
\hline Axilar + Dorso & $1(1,85 \%)$ \\
\hline Plantar + Axilar & $1(1,85 \%)$ \\
\hline Craniofacial & $1(1,85 \%)$ \\
\hline Palmar + Pélvica & $1(1,85 \%)$ \\
\hline Facial + Craniofacial + Abdome & $1(1,85 \%)$ \\
\hline $\begin{array}{c}\text { Palmar + Plantar+ Axilar }+ \\
\text { Facial }\end{array}$ & $1(1,85 \%)$ \\
\hline $\begin{array}{c}\text { Palmar + Plantar + Axilar + } \\
\text { Facial + Dorsal }\end{array}$ & $1(1,85 \%)$ \\
\hline
\end{tabular}

Fonte: Elaborada pelos autores.

De acordo com a gravidade da HP, a maioria dos estudantes se enquadrou no grau $2(66,67 \%)$, seguido respectivamente dos graus $1(20,37 \%), 3(11,11 \%)$ e $4(1,85 \%)$. Com relação à autoavaliação sobre a QvD desses portadores: 7 (12,96\%) a classificaram como excelente, $15(27,79 \%)$ como muito boa, 21 $(38,89 \%)$ como boa, 10 (18,52\%) como ruim e 1 (1,85\%) como muito ruim (Tabela 2 ).

A correlação da gravidade e da QdV dos portadores de HP evidenciou que, no grau 1 da doença, 6 (54,54\%) estudantes

\begin{tabular}{|c|c|c|c|c|}
\hline \multicolumn{5}{|c|}{$\begin{array}{l}\text { Distribuição estatística de uma amostra de } 54 \text { acadêmicos portadores de HP do curso de Medicina } \\
\text { de uma universidade privada de Aracaju/SE, no ano de 2017, quanto à QdV e HDSS }\end{array}$} \\
\hline HDSS & Frequência & Porcentagem & Porcentagem válida & Porcentagem acumulativa \\
\hline Grau 1 & 11 & 20,37 & 20,37 & 20,37 \\
\hline Grau 2 & 36 & 66,67 & 66,67 & 87,04 \\
\hline Grau 3 & 6 & 11,11 & 11,11 & 98,15 \\
\hline Grau 4 & 1 & 1,85 & 1,85 & 100 \\
\hline Total & 54 & 100 & 100 & \\
\hline QdV & Frequência & Porcentagem & Porcentagem válida & Porcentagem acumulativa \\
\hline Excelente & 7 & 12,96 & 12,96 & 12,96 \\
\hline Muito boa & 15 & 27,78 & 27,78 & 40,74 \\
\hline Boa & 21 & 38,89 & 38,89 & 79,63 \\
\hline Ruim & 10 & 18,52 & 18,52 & 98,15 \\
\hline Muito ruim & 1 & 1,85 & 1,85 & 100 \\
\hline Total & 54 & 100 & 100 & \\
\hline
\end{tabular}

Fonte: Elaborada pelos autores. 
a consideraram excelente; 4 (36,36\%), muito boa; e 1 (9,09\%), boa. No grau 2 , somente $1(2,78 \%)$ acadêmico a avaliou como excelente; 11 (30,55\%), como muito boa; 19 (52,78\%), como boa; e 5 (13,89\%), como ruim. No grau 3, 1 (16,70\%) aluno considerou a QdV boa; 4 (66,60\%), ruim; e 1 (16,70\%), muito ruim. No grau 4, 1 (100\%) estudante classificou a QdV como ruim.

A QdV referida foi comparada ao escore da HDSS por meio do teste de Shapiro-Wilk para normalidade da distribuição que exibiu valor de $p<0,001$, justificando que a amostra tinha uma distribuição fora da normalidade. $\mathrm{O}$ teste não paramétrico de Kruskal-Wallis foi utilizado e apresentou $p=$ 0,021, sugerindo que a distribuição entre o total dos domínios é diferente entre as categorias de HDSS. Por fim, o teste de Pearson para correlação das variáveis - utilizando como variável de agrupamento os grupos HDSS 1, HDSS 2 e HDSS 3 ou 4 - foi aplicado e apresentou $p<0,01$. Assim, pode-se inferir que níveis maiores de QdV são inversamente proporcionais aos escores da HDSS.

Na Tabela 3, estão representados os valores da distribuição dos domínios social, pessoal, emocional e em condições especiais, e a Figura 2 apresenta as frequências das pontuações do domínio total e a consequente a soma de todos os domínios de acordo com o questionário de QdV de Campos et al. ${ }^{4}$

\section{TABELA 3}

Distribuição estatística de uma amostra de 54 acadêmicos portadores de HP do curso de Medicina de uma universidade privada de Aracaju/SE, no ano de 2017, quanto aos domínios funcionais de Campos et al. ${ }^{4}$

\begin{tabular}{ccccccc} 
Domínios & Número & Mínimo & Máximo & Média & $\begin{array}{c}\text { Erro } \\
\text { desvio }\end{array}$ \\
\hline Funcional-social & 54 & 8 & 39 & 20,15 & 8,32 \\
\hline Pessoal & 54 & 3 & 15 & 6,91 & 3,88 \\
\hline Emocional & 54 & 2 & 10 & 5,24 & 2,64 \\
\hline Especial & 54 & 7 & 35 & 21,33 & 7,08
\end{tabular}

Fonte: Elaborada pelos autores.

Utilizou-se o teste de Shapiro-Wilk para testar a normalidade do domínio total e constatou-se um $\mathrm{p}=0,449$. Por meio da escala de domínios de Campos et al. ${ }^{4}$, observaram-se as seguintes médias de escore de gravidade: 42 para HDSS 1, 54 para HDSS 2, 70 para HDSS 3 e 74 para HDSS 4. Com o teste de Anova para avaliação dos subescores do domínio total, obtiveram-se valores estatisticamente diferentes entre as categorias de HDSS $(p=0,018)$. Os maiores graus de HP se correlacionaram com níveis mais elevados no questionário de Campos et al. ${ }^{4}$, o que significa uma pior $\mathrm{QdV}$.
Figura 2

Distribuição da pontuação para a QdV referente ao domínio total do questionário de Campos et al. ${ }^{4}$ dada por uma amostra de 54 acadêmicos do curso de Medicina de uma universidade privada de Aracaju/SE, no ano de 2017

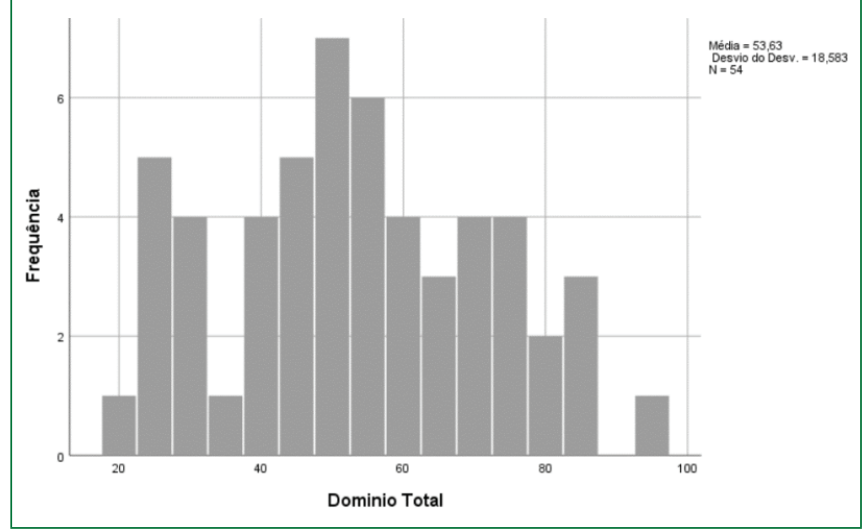

Fonte: Elaborada pelos autores.

\section{DISCUSSÃO}

A HP é descrita por Moya et al. ${ }^{24}$ com uma prevalência na população mundial maior que 1\%. No Brasil, Felini, Demarchi, Fistarol, Matiello e Delorenze ${ }^{13}$ identificaram em Blumenau que $9,0 \%$ dos indivíduos possuíam HP. Em uma amostra de estudantes de medicina, Westphal et al. ${ }^{10}$ notificaram que 5,5\% eram portadores da doença. Lima et al. ${ }^{17}$ mostraram que a HP foi relatada em 14,76\% também em discentes de medicina. Há evidências de que a incidência da HP se alterar conforme as características culturais e climáticas e até mesmo por causa das diferenças de metodologias utilizadas ${ }^{25}$. No presente estudo, a frequência de HP encontrada em acadêmicos de medicina foi de $18 \%$, semelhante entre homens e mulheres, o que corrobora os estudos mais recentes em que observa uma prevalência de até $16,8 \%$, sem diferença entre os sexos ${ }^{10,11,13,17,26-31}$. Ressalta-se, portanto, uma alta prevalência nesses acadêmicos que necessitam ter contato com outras pessoas durante o exame clínico em momentos de estresse devido à supervisão do seu tutor.

A prevalência de HP foi maior na cor branca, seguida das cores parda e negra, o que corrobora outras pesquisas ${ }^{32,33}$. A faixa etária média foi de 21,57 anos nos portadores de $\mathrm{HP}$, acometendo indivíduos mais jovens, resultado similar ao estudo de Lima et al. ${ }^{17}$ com média de 23 anos. Em Park, Han, Choi, Kim e Kim ${ }^{34}$, a idade média foi de 28,5 anos; 25,4 anos em Fiorelli et al. ${ }^{3}$ e 17,6 anos Tu et al. ${ }^{27}$. A HP é uma doença crônica, de início em idades precoces, que causa prejuízos nas atividades diárias, sendo importante o seu diagnóstico por ter tratamento curativo. 
Nesta pesquisa, obtiveram-se com frequência relatos de acadêmicos portadores HP que referiram história familiar positiva, resultado similar aos estudos de outros autores ${ }^{10,13}$. O período de início dos sintomas de hiperidrose predominou na adolescência, seguido da infância e da fase adulta, como observado por Moraites, Vaughn e Hill ${ }^{30}$, em que as manifestações clínicas começavam entre 14 e 25 anos de idade, e Tu et al..$^{27}$, entre 4 e 22 anos. Apesar de os primeiros sintomas surgirem na infância ou adolescência, a maioria dos portadores de HP só procura ajuda na fase adulta, isto é, eles convivem boa parte da sua vida com essa comorbidade e seus transtornos até terem autonomia e conhecimento para buscar tratamento ${ }^{4,5}$. Uma maior compreensão científica dos acadêmicos de medicina sobre as características clínicas de doenças crônicas deveria favorecer o diagnóstico e tratamento precoces da HP, porém essa afecção é pouco discutida na grade médica curricular.

O perfil clínico dos acadêmicos de medicina portadores de HP segue os critérios descritos por Hornberger et al. ${ }^{21}$, Felini, Demarchi, Fistarol, Matiello e Delorenze ${ }^{13}$ e Lima et al. ${ }^{17}$. Dados mostram que o diagnóstico da HP é fácil e eminentemente clínico.

A região palmar foi a área mais referida pelos alunos. Os resultados discordam do estudo de Lear, Kessler, Solish e Glaser $^{35}$, em que a região axilar era em $73 \%$ o sítio mais afetado, seguido da palmar $(45,9 \%)$ e plantar $(41,1 \%)$. Esse resultado corrobora os achados de Westphal et al. ${ }^{10}$ e Lima et al. ${ }^{17}$, que consideraram, respectivamente, a região palmar o local mais frequentemente acometido em $36 \%$ e 39,62\% dos indivíduos. A incapacidade de realizar atividades simples como apertar a mão de outras pessoas ou executar o manuseio prático exigido para a formação médica, por causa sobretudo da hiperidrose palmar, pode causar situações constrangedoras e estressantes.

A descrição de gravidade da HP pelos estudantes de medicina mostrou o predomínio da hiperidrose leve a moderada. A presença de HP nos graus 3 e 4, embora significativamente menor, apresentou maior gravidade, dificultando consideravelmente os afazeres dos acadêmicos. De acordo com Weber et al. $^{36}$, a maioria dos pacientes com HP não se consideram tímidos ou o são pelos que estão à sua volta. A ansiedade $\mathrm{e}$ hiperidrose têm uma relação complicada: apesar de esta manifestar-se espontaneamente, ela é exacerbada por aquela ${ }^{37,38}$. $\mathrm{O}$ estresse em estudantes de medicina é constante pelo fato de eles enfrentarem situações desgastantes físicas e emocionais durante as atividades acadêmicas ${ }^{39}$. A sudorese excessiva, portanto, pode ser interpretada pelos pacientes e preceptores como ansiedade e falta de autoconfiança, o que pode rotular os acadêmicos portadores de HP como ansiosos e inseguros.
A maioria significativa dos acadêmicos portadores de HP classificou a própria $\mathrm{QdV}$ como excelente, muito boa ou boa. Embora, 18,52\% e 1,85\%, respectivamente, tenham classificado a própria $\mathrm{QdV}$ como ruim e muito ruim, verificou-se que as piores descrições de QdV são inversamente proporcionais aos escores da HDSS. A HP é considerada por alguns pesquisadores como doença de baixo risco, porém acarreta aos seus portadores profundo constrangimento social, psíquico, profissional e emocional ${ }^{21,40}$, com impactos na $\mathrm{QdV}^{41} \mathrm{e}$ nas atividades diárias desses indivíduos ${ }^{42,43}$. Embora a HP seja considerada uma doença crônica benigna, um percentual importante dos acadêmicos de medicina classificou a própria $\mathrm{QdV}$ relacionada à $\mathrm{HP}$ como ruim e muito ruim, o que mostra que, a depender do grau de intensidade, a doença interfere negativamente na QdV. Os portadores dessa afecção em graus leve e moderado declararam não ter interferência no seu desempenho diário, o que mostra a importância de se avaliar o grau de comprometimento da HP nos estudos populacionais, assim como relacioná-la com a própria $\mathrm{QdV}$ para melhor direcionar a terapêutica.

Nas atividades que retratam a QdV, constam, no domínio funcional descrito pelos acadêmicos, os piores preditores (ruim e muito ruim) para dançar socialmente e apertar mãos. No domínio pessoal e emocional, as maiores dificuldades encontradas eram segurar as mãos do parceiro(a) e justificar o suor excessivo. No domínio de condições especiais, as piores respostas enquadram-se nos seguintes contextos: estar em ambientes fechados ou quentes, vivenciar situações de preocupação ou tensão e momentos que precedem uma prova e falar em grupo. Os dados encontrados nessa população específica foram semelhantes aos de outros estudos, que também avaliaram a QdV em portadores de HP, independentemente do seu labor, e confirmaram que a doença provoca desconforto intenso e baixa autoestima, além de impacto negativo na QdV nos ambientes social e profissional ${ }^{44-46}$.

Pode-se afirmar que o impacto na QdV é diretamente proporcional ao grau da HP, uma vez que, em graus moderados e graves (3 e 4), notou-se um comprometimento importante na QdV dos pacientes. Deve-se considerar ainda que, por conta da segregação social advinda da própria doença, os indivíduos portadores de HP silenciam quanto ao acometimento dos seus efeitos nocivos, causando impacto significante no desempenho de suas vidas pessoal e profissional, sendo considerados indicadores de saúde ${ }^{10,47}$. Alguns portadores de HP omitem a doença e não procuram tratamento porque querem evitar qualquer constrangimento relacionado a uma afecção que ainda é pouco conhecida.

Embora seja uma doença crônica, com prevalência significativa, de início nas primeiras décadas de vida, com interferên- 
cia negativa biopsicossocial, os próprios doentes e, às vezes, os profissionais da área de saúde desconhecem a existência desse agravo como doença. Esses fatos demostram a necessidade de uma maior divulgação da HP mediante pesquisas cientificas para um melhor conhecimento da sua prevalência e das consequências tanto no meio acadêmico quanto na população em geral.

\section{CONCLUSÃO}

A prevalência de HP foi de $18 \%$ nos acadêmicos de medicina de uma universidade privada de Aracaju/SE, sem diferença entre os sexos e com predomínio das cores branca e parda. A doença ocorreu principalmente em sítios combinados, como palmoplantar, sendo a região palmar a mais acometida. Os sintomas iniciaram-se, principalmente, durante a infância e adolescência, com prejuízo importante nas atividades diárias pessoais e laborativas, e exacerbação do suor com situações de estresse. A gravidade da HP mais comum foi a moderada. Sobre o impacto negativo na $\mathrm{QdV}$, apontam-se principalmente os graus mais avançados da doença que dificultam as atividades acadêmicas. É importante a inclusão do conhecimento da HP no currículo médico para maior divulgação da doença, de modo a permitir o diagnóstico precoce e relacionado à intensidade da sudorese na sua abordagem terapêutica.

\section{LIMITAÇÕES}

O estudo realizado apresentou como limitação uma amostragem restrita a uma universidade, por ser a única com o curso de Medicina de Aracaju/SE. Embora o cálculo amostral de 300 estudantes, tenha sido superior ao valor previsto pela fórmula de Barbetta, estudos posteriores devem ser realizados para melhor confirmar os resultados do presente trabalho, analisando o perfil dos portadores e não portadores de HP.

\section{REFERÊNCIAS}

1. Strutton DR, Kowalski JW, Glaser DA, Stang PE. US prevalence of hyperhidrosis and impact on individuals with axillary hyperhidrosis: results from a national survey. J Am Acad Dermatol. 2004 Aug;51(2):241-248.

2. Cavalcante JF, Araújo CA, Nettol MX, da Costa FE, Diniz Filho FF, Ada CM. Effects of endoscopic sympathicotomy in carotid and vertebral arteries in the surgical treatment of primary hyperhidrosis. Acta Cir Bras. 2005;20(Suppl 1):146-151.

3. Fiorelli RKA, Elliot LG, Alvarenga RMPA, Morard MRS, Almeida CRA, Fiorelli SKA, et al. Avaliação do impacto na qualidade de vida de pacientes portadores de hiperidrose primária submetidos à simpatectomia videotoracoscópica. Meta Aval. 2011;3(7):1-24
4. Campos JRM, Kauffman P, Werebe EC, Andrade FLO, Kuzniek S, Wolosker N, et al. Questionnaire of quality of life in patients with primary hyperhidrosis. J. Pneumologia. 2003 Aug;29(4):178-181.

5. Cardoso PO, Rodrigues KCL, Mendes KM, Petroianu A, Resende M, Alberti LR. Avaliação de pacientes submetidos a tratamento cirúrgico de hiperidrose palmar quanto à qualidade de vida e ao surgimento de hiperidrose compensatória. Rev. Col. Bras. Cir. 2009;36(1):14-18.

6. Cohen JL, Cohen G, Solish N, Murray CA. Diagnosis, impact, and management of focal hyperhidrosis: treatment review including botulinum toxin therapy. Facial Plast Surg Clin North Am. 2007 Feb;15(1):17-30.

7. Solish N, Wang R, Murray CA. Evaluating the patient presenting with hyperhidrosis. Thorac Surg Clin. 2008 May;18(2):133-140.

8. Gontijo GT, Gualberto GV, Madureira NAB. Axillary hyperhidrosis treatment update. Surg Cosmet Dermatol. 2011;3(2):147-151.

9. Solish N, Bertucci V, Dansereau A, Hong HC, Lynde C, Lupin $\mathrm{M}$, et al. A comprehensive approach to the recognition, diagnosis, and severity-based treatment of focal hyperhidrosis: recommendations of the Canadian Hyperhidrosis Advisory Committee. Dermatol Surg. 2007 Aug;33(8):908923.

10. Westphal FL, Surgeon T, Carvalho MAN, Lima LC, Carvalho BCN, Padilla R, et al. Prevalência de hiperidrose entre estudantes de medicina. Rev. Col. Bras. Cir. 2011;38(6):392397.

11. Augustin M, Radtke MA, Herberger K, Kornek T, Heigel H, Schaefer I. Prevalence and disease burden of hyperhidrosis in the adult population. Dermatology. 2013;227(1):10-13.

12. Li X, Chen R, Tu YR, Lin M, Lai FC, Li YP, et al. Epidemiological survey of primary palmar hyperhidrosis in adolescents. Chin Med J. 2007 Dec 20;120(24):2215-2217.

13. Felini R, Demarchi AR, Fistarol ED, Matiello M, Delorenze LM. Prevalência de hiperidrose em uma amostra populacional de Blumenau - SC, Brasil. An. Bras. Dermatol. 2009; 84(4):361-366.

14. Wolosker N, Munia MA, Kauffman P, Campos JR, Yazbek G, Puech-Leão P. Is gender a predictive factor for satisfaction among patients undergoing sympathectomy to treat palmar hyperhidrosis? Clinics. 2010 June;65(6):583-586.

15. Lessa LR, Fontenelle LF. Toxina botulínica como tratamento para fobia social generalizada com hiperidrose. Rev. Psiquiatr. Clín. 2011;38(2):84-86.

16. Hamm H. Impact of hyperhidrosis on quality of life and its assessment. Dermatol Clin. 2014 Oct;32(4):467-476. 
17. Lima SO, Aragão JF, Machado Neto J, Almeida KB, Menezes LM, Santana VR. Research of primary hyperhidrosis in students of medicine of the State of Sergipe, Brazil. An Bras Dermatol. 2015;90(5):661-665.

18. Dias LI, Miranda EC, Toro IF, Mussi RK. Relationship between anxiety, depression and quality of life with the intensity of reflex sweating after thoracoscopic sympathectomy for treatment of primary hyperhidrosis. Rev Col Bras Cir. 2016 Sep-Oct;43(5):354-359.

19. Muthusamy A, Gajendran R, Ponnan S, Thangavel D, Rangan V. A study on the impact of hyperhidrosis on the quality of life among college students. J Clin Diagn Res. 2016;10(6):CC08-10.

20. Cetindag IB, Boley TM, Webb KN, Hazelrigg SR. Long-term results and quality-of-life measures in the management of hyperhidrosis. Thorac Surg Clin. 2008 May;18(2):217-222.

21. Hornberger J, Grimes K, Naumann M, Glaser DA, Lowe NJ, Naver H, et al. Recognition, diagnosis, and treatment of primary focal hyperhidrosis. J Am Acad Dermatol. 2004 Aug;51(2):274-286.

22. Barbetta PA. Estatística aplicada às ciências sociais. 7. ed. Florianópolis: Editora da UFSC; 2010.

23. Amir M, Arish A, Weinstein Y, Pfeffer M, Levy Y. Impairment in quality of life among patients seeking surgery for hyperhidrosis (excessive sweating): preliminary results. Isr J Psychiatry Relat Sci. 2000;37(1):25-31.

24. Moya J, Ramos R, Morera R, Villalonga R, Perna V, Macia I, et al. Thoracic sympathicolysis for primary hyperhidrosis: a review of 918 procedures. Surg Endosc. 2006 Apr;20(4):598-602.

25. Cerfolio RJ, De Campos JR, Bryant AS, Connery CP, Miller DL, DeCamp MM, et al. The Society of Thoracic Surgeons expert consensus for the surgical treatment of hyperhidrosis. Ann Thorac Surg. 2011 May;91(5):1642-1648.

26. Stori Jr. WS, Coelho MS, Guimarães PSF, Bergonse NN, Pizarro LDV. Thoracic sympathetic block by clamping for treatment of hyperhidrosis. An. Bras. Dermatol. 2006 Oct; 81(5):425-432.

27. Tu YR, Li X, Lin M, Lai FC, Li YP, Chen JF, et al. Epidemiological survey of primary palmar hyperhidrosis in adolescent in Fuzhou of People's Republic of China. Eur J Cardiothorac Surg. 2007 Apr;31(4):737-739.

28. Stefaniak T, Tomaszewski KA, Proczko-Markuszewska M, Idestal A, Royton A, Abi-Khalil C. Is subjective hyperhidrosis assessment sufficient enough? Prevalence of hyperhidrosis among young polish adults. J Dermatol. 2013 Oct;40(10):819-823.
29. Fujimoto T, Kawahara K, Yokozeki H. Epidemiological study and considerations of primary focal hyperhidrosis in Japan: from questionnaire analysis. J Dermatol. 2013 Nov;40(11):886-890.

30. Moraites E, Vaughn OA, Hill S. Incidence and prevalence of hyperhidrosis. Dermatol Clin. 2014 Oct;32(4):457-465.

31. Lima SO, Santana VR. The prevalence of hyperhidrosis worldwide. In Loureiro M, Campos J, Wolosker N, Kauffman P, editors. Hyperhidrosis. Chan: Springer; 2018. p. 33-38.

32. Buraschi J. Videothoracoscopic sympathicolysis procedure for primary palmar hyperhidrosis in oliveichildren and adolescents. Arch Argent Pediatr. 2008 Feb;106(1):32-35.

33. Gelbard CM, Epstein H, Hebert A. Primary pediatric hyperhidrosis: a review of current treatment options. Pediatr Dermatol. 2008 Nov-Dec;25(6):591-598.

34. Park EJ, Han KR, Choi H, Kim DW, Kim C. An epidemiological study of hyperhidrosis patients visiting the Ajou University Hospital hyperhidrosis center in Korea. J Korean Med Sci. 2010;25(5):772-775.

35. Lear W, Kessler E, Solish N, Glaser DA. An epidemiological study of hyperhidrosis. Dermatol Surg. 2007 Jan;33:S69-75.

36. Weber A, Heger S, Sinkgraven R, Heckmann M, Elsner P, Rzany B. Psychosocial aspects of patients with focal hyperhidrosis. Marked reduction of social phobia, anxiety and depression and increased quality of life after treatment with botulinum toxin A. Br J Dermatol. 2005 Feb;152(2):342-345.

37. Bragança GM, Lima SO, Pinto Neto AF, Marques LM, Melo EV, Reis FP. Evaluation of anxiety and depression prevalence in patients with primary severe hyperhidrosis. An Bras Dermatol. 2014 Mar-Apr;89(2):230-235.

38. Schneier FR, Heimberg RG, Liebowitz MR, Blanco C, Gorenstein LA. Social anxiety and functional impairment in patients seeking surgical evaluation for hyperhidrosis. Compr Psychiatry. 2012 Nov;53(8):1181-1186.

39. Santos FS, Maia CRC, Faedo FC, Gomes GPC, Nunes ME, Oliveira MVM. Estresse em estudantes de cursos preparatórios e de graduação em Medicina. Rev. Bras. Educ. Méd. 2017 Jun;41(2):194-200.

40. De Campos JR, Kauffman P, Werebe EC, Andrade Filho LO, Kusniek S, Wolosker N, et al. Quality of life, before and after thoracic sympathectomy: report on 378 operated patients. Ann Thorac Surg. 2003 Sep;76(3):886-891.

41. Vanderhelst E, De Keukeleire T, Verbanck S, Vincken W, Noppen M. Quality of life and patient satisfaction after video-assisted thoracic sympathicolysis for essential hyperhidrosis: a follow-up of 138 patients. J Laparoendosc Adv Surg Tech A. 2011 Dec;21(10):905-909. 
42. Haider A, Solish N. Focal hyperhidrosis: diagnosis and management. CMAJ. 2005;172(1):69-75.

43. Wang R, Solish N, Murray CA. Primary focal hyperhidrosis: diagnosis and management. Dermatol Nurs. 2008 Dec;20(6):467-470.

44. Sampaio GAA, Almeida ART, Saliba AFN, Queiroz NPL. Hiperidrose inframamária: caracterização clínica e gravimétrica. Surg Cosmet Dermatol. 2013;5(2):146-149.

45. Henriques M, Costa J. Botulinum toxin type A iontophoresis in palmar hyperhidrosis. Revista da Sociedade Portuguesa de Medicina Física e de Reabilitação. 2014:26(2):36-40.

46. Souza LG, Salomão Júnior A, Mattos RA, Steiner D, Signor KC, Michalany AO. Radiofrequência bipolar no tratamento da hiperhidrose axilar: um estudo-piloto. Surg Cosmet Dermatol 2015;7(3): 228-231.

47. Wolosker N, Campos JRM, Kauffman P, Munia MA, Neves $S$, Jatene FB, et al. The use of oxybutynin for treating facial hyperhidrosis. An Bras Dermatol. 2011 May-June;86(3):451-456.

\section{CONTRIBUIÇÃO DOS AUTORES}

Sônia Oliveira Lima participou da concepção, da coleta de dados, da análise dos dados, da redação e correção final do trabalho. Maria Tainar Barbosa de Almeida e Fernanda Rodrigues Rocha participaram da concepção, da coleta de dados, da análise dos dados e da redação final do trabalho. Rafael Silva Santos participou da análise dos dados e da redação final do trabalho. Renata Lima Batalha de Andrade participou da coleta de dados e da redação final do trabalho. Carla Viviane Freitas de Jesus participou da análise dos dados, da redação e correção final do trabalho.

\section{CONFLITO DE INTERESSES}

Os autores declaram não haver conflito de interesses neste estudo.

\section{ENDEREÇO PARA CORRESPONDÊNCIA}

Sonia Oliveira Lima

Mestre e Doutora em Medicina pela Universidade de São Paulo. Professora Titular da Pós-Graduação em Saúde e Ambiente da Universidade Tiradentes.

Avenida Murilo Dantas, 300

Aracaju - Sergipe - SE

CEP: $49032-490$

E-mail: sonialima.cirurgia@gmail.com 